EPJ Web of Conferences 71, 00025 (2014)

DOI: $10.1051 /$ epjconf / 20147100025

(C) Owned by the authors, published by EDP Sciences, 2014

\title{
Measurements of Higgs Boson Properties using the ATLAS De- tector
}

\author{
Tingting $\mathrm{Cao}^{1, \mathrm{a}}$
}

For the ATLAS collaboration

${ }^{1}$ Department of Physics, Southern Methodist University

\begin{abstract}
Measurements are presented of the production properties, couplings and spinparity of the recently discovered Higgs-like boson using the decays into boson pairs, $\gamma \gamma$, $Z Z^{*} \rightarrow 4 \ell$ and $W W^{*} \rightarrow \ell v \ell v$. The results shown here are based on the complete $p p$ collision data sample recorded by the ATLAS experiment at the Large Hadron Collider (LHC) at centre-of-mass energies of $\sqrt{\mathrm{s}}=7 \mathrm{TeV}$ and $\sqrt{\mathrm{s}}=8 \mathrm{TeV}$, and correspond to an integrated luminosity of about $25 \mathrm{fb}^{-1}$. Evidence for Higgs boson production through vector-boson fusion is reported. Results of combined fits probing Higgs boson couplings to fermions and bosons, as well as anomalous contributions of loop-induced production and decay modes, are presented. The spin-parity hypothesis $J^{P}=0^{+}$, predicted for the Standard Model Higgs boson, is compared to alternative hypotheses $\left(J^{P}=0^{-} 1,1^{+}, 1^{-}\right.$ and $2^{+}$). All measurements are consistent with the Standard Model expectations of the Higgs boson.
\end{abstract}

\section{Introduction}

The discovery of a new particle with a mass of about $125 \mathrm{GeV}$ in the search for the Standard Model (SM) Higgs boson at the CERN Large Hadron Collider (LHC) was reported in July 2012 by the ATLAS and CMS Collaborations[1]. The present experimental challenge is to compare its properties with those predicted by the Standard Model. This proceeding presents measurements of the main properties of the newly observed particle, including its mass, production strengths and couplings to fermions and bosons, as well as spin and parity, using diboson final states: $\gamma \gamma, Z Z^{*} \rightarrow 4 \ell$ and $W W^{*} \rightarrow \ell v \ell v$.

The results reported here are based on the data samples recorded with the ATLAS detector in 2011 (at $\sqrt{\mathrm{s}}=7 \mathrm{TeV}$ ) and 2012 (at $\sqrt{\mathrm{s}}=8 \mathrm{TeV}$ ), corresponding to integrated luminosities of $4.7 \mathrm{fb}^{-1}$ and $20.7 \mathrm{fb}^{-1}$, respectively.

\section{Contributing channels}

Under the hypothesis that the newly discovered boson is a Standard Model Higgs boson, we consider the following production processes: gluon fusion $\left(g g \rightarrow H\right.$, denoted ggF), vector-boson fusion $\left(q q^{\prime} \rightarrow\right.$ $q q^{\prime} H$, denoted VBF) and Higgs-strahlung $\left(q q^{\prime} \rightarrow W H, Z H\right.$, denoted $W H / Z H$ or, jointly, $\left.V H\right)$. The

\footnotetext{
a e-mail: tingting.cao@cern.ch
} 
small contribution from the associated production with a $t \bar{t}$ pair $(g g / q q \rightarrow t t H$, denoted $t t H)$ is taken into account in the $\gamma \gamma$ and $Z Z^{*}$ analyses. These are used for the comparison of data and the theoretical expectation.

The diphoton invariant mass distribution, after applying our event selection[2] on the full data sample is shown in Fig. 1-(a). At the maximum deviation from the background-only expectation, which occurs for $m_{H} \sim 126.5 \mathrm{GeV}$, the local significance of the observed peak is $7.4 \sigma$ for the combined $7 \mathrm{TeV}$ and $8 \mathrm{TeV}$ data. This observation can be compared with $4.3 \sigma$ significance expected from SM Higgs boson production at this mass. Within the range of $m_{H}=126.5 \pm 4 \mathrm{GeV}$, there are about $1.7 \times 10^{4}$ background events and about $10^{3}$ signal events. A discovery-level signal is achieved in the $\gamma \gamma$ channel alone.

The reconstructed four-lepton mass spectrum after all analysis selections [3] is shown in Fig. 1-(b). The data are compared to the (scaled) expected Higgs boson signal for $m_{H}=124.3 \mathrm{GeV}$ and to the estimated backgrounds. At the maximum deviation from the background-only expectation (occurring at $m_{H}=124.3 \mathrm{GeV}$ ), the local significance of the observed peak is $6.6 \sigma$ for the combined $7 \mathrm{TeV}$ and 8 $\mathrm{TeV}$ data, to be compared with $4.4 \sigma$ expected from SM Higgs boson production at this mass. Within the range of $m_{H}=124.3 \pm 3.7 \mathrm{GeV}$, there are 8 background events and 22 signal events. This result establishes a discovery-level signal in the $Z Z^{*} \rightarrow 4 \ell$ channel alone. The invariant mass of the $W W^{*}$

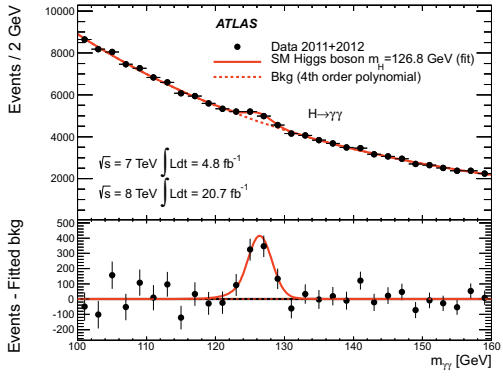

(a)

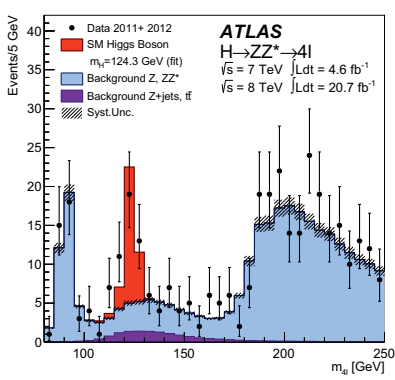

(b)

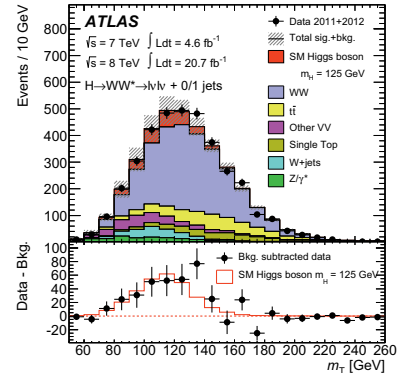

(c)

Figure 1. (a) Invariant mass distribution of diphoton candidates; (b) the four-lepton invariant mass, $\mathrm{m}_{4 \ell}$; (c) the transverse mass distributions of $\ell v \ell v$ after all event selection is applid. All distributions are from a combined 7 $\mathrm{TeV}$ and $8 \mathrm{TeV}$ data sample[1].

system cannot be reconstructed due to a lack of measurements of the momentum of neutrinos from $W$ decay. Transverse mass $\left(m_{T}\right)$ is used instead[4]. The transverse mass distribution after the full selection for $N_{\text {jet }} \leq 1$ final states is shown in the Fig. 1-(c). The region $m_{T}>150 \mathrm{GeV}$ is depleted of signal contribution; the level of agreement of the data with the expectation in this region, which is different from those used to normalize the backgrounds, illustrates the quality of the background estimates. The expected numbers of signal and background events at $8 \mathrm{TeV}$ are 1036 background events and 152 signal events. An excess of events relative to the background-only expectation is observed in the data, with the maximum deviation $(4.1 \sigma)$ occurring at $m_{H}=140 \mathrm{GeV}$. For $m_{H}=$ $125.5 \mathrm{GeV}$, a significance of $3.8 \sigma$ is observed, compared with an expected value of $3.8 \sigma$ for a SM Higgs boson. 


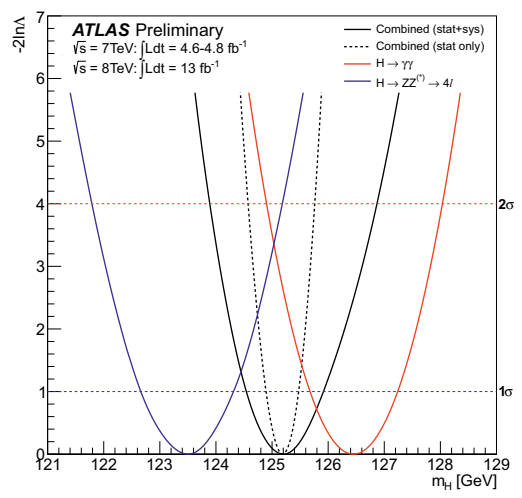

(a)

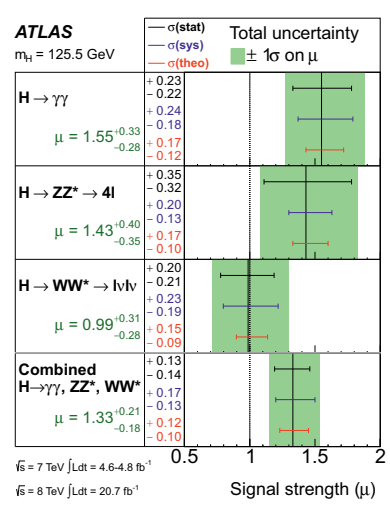

(b)
Figure 2. (a) The profile likelihood ratio $-2 \ln \Lambda\left(m_{H}\right)$ as a function of $m_{H}$ for each channel and their combination. The signal strengths $\mu_{\gamma \gamma}$ and $\mu_{4 l}$ is allowed to vary independently. (b) The measured production strengths for a Higgs boson of mass $m_{H}=125.5 \mathrm{GeV}$, normalized to the SM expectations, for diboson final states and their combination.

\section{Mass and signal strength}

The mass of the new particle is measured from the data using the two channels with the best mass resolution, $\gamma \gamma$ and $Z Z^{*} \rightarrow 4 \ell$. Fits to the mass spectra yield $m_{H}=126.8 \pm 0.2$ (stat) \pm 0.7 (sys) GeV and $m_{H}=124.3_{-0.5}^{+0.6}$ (stat) ${ }_{-0.3}^{+0.5}$ (sys) GeV. This result assumes the new boson is a Higgs particle.

To derive a combined mass measurement, the profile likelihood ratio $\Lambda\left(m_{H}\right)$ is used, and the ratios of the cross sections for the various production modes for each channel are fixed to the SM values. The likelihood function for the fit to the Higgs mass is shown on Fig. 2-(a). The combined mass is measured to be:

$$
m_{H}=125.5 \pm 0.2 \text { (stat) }{ }_{-0.6}^{+0.5}(\mathrm{sys}) \mathrm{GeV} .
$$

The Higgs boson production strength parameter $\mu$, i.e., the ratio of the observed rate to the Standard Model expectation, is determined from a fit to the data using the profile likelihood ratio $\Lambda(\mu)$ for a fixed mass hypothesis corresponding to the measured value $m_{H}=125.5 \mathrm{GeV}$. Uncertainties are incorporated into the likelihood function assuming Gaussian distribution of the systematic errors.

The results are shown in Fig. 2-(b), where the production strengths for the $\gamma \gamma, Z Z^{*} \rightarrow 4 \ell$ and $W W^{*} \rightarrow \ell v \ell v$ channels are presented for the main analysis categories, together with their overall combination. All results for the individual channels and for their combinations are consistent with the Standard Model predictions for a Higgs boson. The overall signal production strength is measured to be:

$$
\mu=1.33 \pm 0.14 \text { (stat) } \pm 0.15 \text { (sys) }
$$

\section{Production mechanism and couplings}

Vector-boson-mediated processes VBF and VH are sensitive to other production mechanisms. Comparison of the relative signal strengths of vector-boson-mediated processes and that of gluon-mediated processes is performed for all three channels. Each channel is found to be compatible with the Standard Model.

Higgs boson couplings to fermions and other bosons are present in both the production and decy process. The Standard Model makes specific predictions about the couplings, and the relationship of different production and decay processes. Measuring the couplings of the Higgs boson in ATLAS 
EPJ Web of Conferences

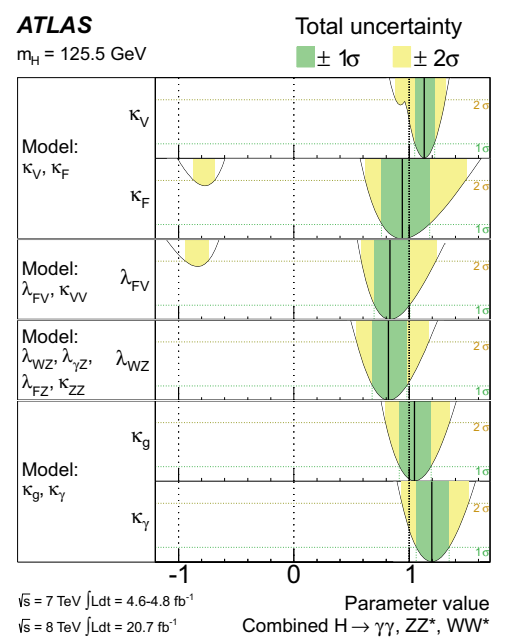

Figure 3. Summary of the measurements of the coupling scale factors for a Higgs boson with mass $\mathrm{mH}=125.5 \mathrm{GeV}$.

data allows us to test the compatibility of production and decay with the predictions of the Standard Model. In order to test different Standard Model predictions, we group couplings using different scenarios. For instance, we can assume common couplings for fermions and vector bosons: $\kappa_{F}$ and $\kappa_{V}$. In this case, the data prefers the couplings with both $k a p p a_{F}$ and $k a p p a_{V}$ larger than zero, which is consistent with the Standard Model predictions. The two-dimensional compatibility of the Standard Model predictions with the best-fit value is $12 \%$. The $68 \%$ Confidence Level (CL) intervals of $\kappa_{F}$ and $\kappa_{V}$, obtained by profiling over the other parameter, are:

$$
\begin{aligned}
& \kappa_{F} \in[0.76,1.18], \\
& \kappa_{V} \in[1.05,1.22] .
\end{aligned}
$$

We can also decompose the $g g \rightarrow H$ and $H \rightarrow \gamma \gamma$ loops into groups containing $t$ and $W$ couplings, or introduce independent $\kappa_{g}$ and $\kappa_{\gamma}$ parameters to allow for Beyond the Standard Model (BSM) loop contributions. Many BSM physics scenarios predict the existence of new heavy particles; these can affect couplings like $\kappa_{g}$ and $\kappa_{\gamma}$. It is assumed that the new particles do not contribute to the Higgs boson width and that the couplings of the known particles to the Higgs boson have SM strength. The results discussed below show the confidence interval of couplings parameters limits if a new particle exists. The two-dimensional compatibility of the SM prediction with the best-fit value is $14 \%$.

\subsection{Results}

The results of the measurements of the coupling scale factors in the various benchmark models for a Higgs boson with mass $125.5 \mathrm{GeV}$ are summarized in Fig. 3. The coupling of a new particle to gauge bosons, $\kappa_{V}$, is constrained by several channels, directly and indirectly, at the $\pm 10 \%$ level. Couplings to fermions with a significance larger than $5 \sigma$ are indirectly observed mainly through the gluon-fusion production process, assuming the loop is dominated by fermion exchange. The ratio of the relative couplings of the Higgs boson to the $\mathrm{W}$ and $\mathrm{Z}$ bosons, $\kappa_{W} / \kappa_{Z}$, is measured to be consistent with unity, as predicted by custodial symmetry[1]. Under the hypothesis that all couplings of the Higgs boson to the known particles are fixed to their SM values, and assuming no BSM contributions to the Higgs boson width, no significant anomalous contributions to the $g g \rightarrow H$ and $H \rightarrow \gamma \gamma$ loops are observed. 
ICNFP 2013

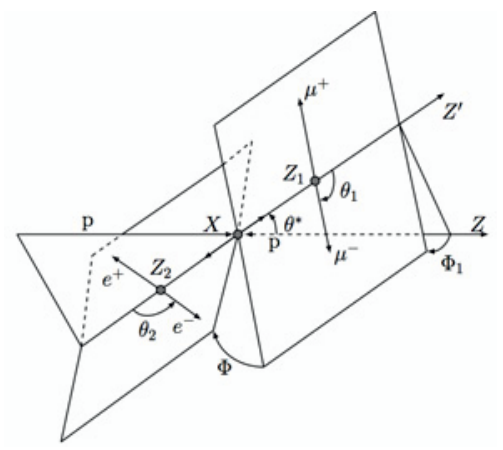

Figure 4. Definition of the production and decay angles in an $X \rightarrow Z Z^{*} \rightarrow 4 \ell$ decay.

\section{Spin and parity}

\subsection{Contributing channels}

ATLAS uses two techniques- Matrix Element Likelihood Analysis (MELA) and Boost Decision Tree (BDT)[5]- to separate the Standard Model expected hypothesis, $J^{P}=0^{+}$, from alternative $J^{P}$ hypotheses in the $X \rightarrow Z Z^{*} \rightarrow 4 \ell$ channel. In this proceeding we focus on the BDT, since both methods have comparable sensitivity. In this channel, there are two decay planes for $Z$ and $Z^{*}$ decaying into pairs of leptons. All the decay and production angles of $X \rightarrow Z Z^{*} \rightarrow 4 \ell$ mode are shown in Fig. 4. These five decay and production angles $\left(\theta^{*}, \phi_{1}, \phi, \theta_{1}\right.$ and $\left.\theta_{2}\right)$, which are used as BDT input variables together with the invariant mass of $Z$ and $Z^{*}$, are sensitive to all possible spins and parity[6].

The Landau-Yang theorem forbids the direct decay of an on-shell spin-1 particle into a pair of photons. The spin-1 hypothesis is therefore strongly disfavored by the observation of the $H \rightarrow \gamma \gamma$ decay. Spin information for this channel can be extracted from the distribution of the absolute value of the cosine of the polar angle $\theta^{*}$ of the photons with respect to the z-axis of the Collins-Soper frame[7]:

$$
\left|\cos \theta^{*}\right|=\frac{\left|\sinh \left(\Delta \eta^{\gamma \gamma}\right)\right|}{\sqrt{1+\left(p_{T}^{\gamma \gamma} / m_{\gamma \gamma}\right)^{2}}} \frac{2 p_{T}^{y^{1}} p_{T}^{y^{2}}}{m_{\gamma \gamma}^{2}},
$$

where $m_{\gamma \gamma}$ and $p_{T}$ are the invariant mass and the transverse momentum of the photon pair, $\Delta \eta^{\gamma \gamma}$ is the separation in pseudo-rapidity of the two photons, and $p_{T}^{y^{1}}, p_{T}^{y^{2}}$ are the transverse momenta of the photons. Two observables, $\left|\cos \theta^{*}\right|$ and $m_{\gamma \gamma}$, are used in the fit to data; $m_{\gamma \gamma}$ provides better separation power between the signal and the background, and $\left|\cos \theta^{*}\right|$ is sensitive to the spin but not sensitive to parity. This channel only contributes to the test of spin- 0 and spin- 2 hypotheses.

The $W W^{*} \rightarrow \ell v \ell v$ channel is sensitive to both spin and parity. At this stage, it is only used to assess the spin of decaying boson. The BDT technique is also used in the $W W^{*} \rightarrow \ell v \ell v$ mode. The variables $m_{l l}, \Delta \Phi_{l l}, p_{T}^{l l}$ and $m_{T}$ are used as inputs to a BDT. Two independent BDT trainings are applied; one separates $J^{P}=0^{+}$from background, while the other separates an alternative $J^{P}$ from background.

\subsection{Statistics}

A test statistics, the ratio of the likelihood of two hypotheses, is used to determine how well we separate two hypotheses using the ATLAS data. The details are discussed below. 
A likelihood function $\mathcal{L}\left(J^{P}, \mu, \theta\right)$ that depends on the spin-parity hypothesis of the signal is constructed as a product of conditional probabilities over binned distributions of the discriminant observables in each channel[5]:

$$
\mathcal{L}\left(J^{p}, \mu, \theta\right)=\prod_{J}^{N_{\text {chann. }}} \prod_{l}^{N_{\text {bins }}} P\left(N_{l, J} \mid \mu_{J} \cdot S_{l, J}^{J^{p}}(\theta)+B_{l, J}(\theta) \times \mathcal{A}_{J}(\theta)\right),
$$

where $\mu_{J}$ represents the nuisance parameter associated with the signal rate in each channel $J$. The symbol $\theta$ represents all other nuisance parameters. Therefore the likelihood function is a product of Poisson distributions, $P$, corresponding to the observation of $N_{l, J}$ events in each bin $l$ of the discriminant observable(s), given the expectations for the signal, $S_{l, J}^{\left(J^{P}\right)}(\theta)$, and for the background, $B_{l, J}(\theta)$. Some of the nuisance parameters are constrained by auxiliary measurements through the functions $\mathcal{A}_{J}(\theta)$.

The test statistic q used to distinguish between the two signal spin-parity hypotheses is based on a ratio of likelihoods:

$$
q=\log \frac{\mathcal{L}\left(J^{P}=0^{+}, \hat{\hat{\mu}}_{0^{+}}, \hat{\hat{\theta}}_{0^{+}}\right)}{\mathcal{L}\left(J_{\text {alt }}^{p}, \hat{\hat{\mu}}_{J_{\text {alt }}^{P}}, \hat{\hat{\theta}}_{J_{\text {alt }}^{P}}\right)},
$$

where $\mathcal{L}\left(J^{P}, \hat{\hat{\mu}}_{J^{P}}, \hat{\hat{\theta}}_{J^{P}}\right)$ is the maximum likelihood estimator, evaluated under either the $0^{+}$or the $J^{P}$ spin-parity hypothesis. The exclusion of the alternative $J^{P}$ hypothesis in favour of the Standard Model $0^{+}$hypothesis is evaluated in terms of the corresponding $\operatorname{CL}_{\mathrm{s}}\left(J_{\text {alt }}^{P}\right)$, defined as:

$$
\mathrm{CL}_{\mathrm{s}}\left(J_{\mathrm{alt}}^{P}\right)=\frac{p_{0}\left(J_{\mathrm{alt}}^{P}\right)}{1-p_{0}\left(0^{+}\right)} \text {. }
$$

\subsection{Results}

The Standard Model Higgs bons is expected to have $J^{P}=0^{+}$. Alternative hypotheses of $J^{p}=0^{-}, 1^{+}$, $1^{-}$and $2^{+}$have so far been tested. Their exclusion confidence levels as well as contributing channels are listed in Table 1. The expected and observed significance to exclude all the alternative hypotheses tested so far are on Fig. 5. The exclusion of all the alternative hypotheses on the plot are above $2 \sigma$. The data prefer spin $0^{+}$compared to spin $0^{-}, 1^{+}, 1^{-}$and $2^{+}$.

Table 1. The confidence level $\mathrm{CL}_{\mathrm{s}}$ for exclusion of $J^{P}$ assignment for the observed boson.

\begin{tabular}{ll|l}
\hline \hline$J^{p}$ & $\mathrm{CL}_{\mathrm{s}}$ & Contributing channels \\
\hline $0^{-}$ & $97.8 \%$ & $H \rightarrow Z Z^{*} \rightarrow 4 \ell$ \\
$1^{+}, 1^{-}$ & $\geq 99.7 \%$ & $H \rightarrow Z Z^{*} \rightarrow 4 \ell, H \rightarrow \gamma \gamma$ \\
$2^{+}$ & $\geq 99.9 \%$ & $H \rightarrow Z Z^{*} \rightarrow 4 \ell, H \rightarrow \gamma \gamma, H \rightarrow W W^{*} \rightarrow \ell \nu \ell v$ \\
\hline \hline
\end{tabular}

\section{Conclusions}

Data recorded by the ATLAS experiment at the LHC in 2011 and 2012, corresponding to an integrated luminosity of up to $25 \mathrm{fb}^{-1}$ at $\sqrt{\mathrm{s}}=7 \mathrm{TeV}$ and $\sqrt{\mathrm{s}}=8 \mathrm{TeV}$, have been analysed to determine several properties of the recently discovered Higgs-like boson using the $\gamma \gamma, Z Z^{*} \rightarrow 4 \ell$ and $W W^{*} \rightarrow \ell v \ell v$ 
ICNFP 2013

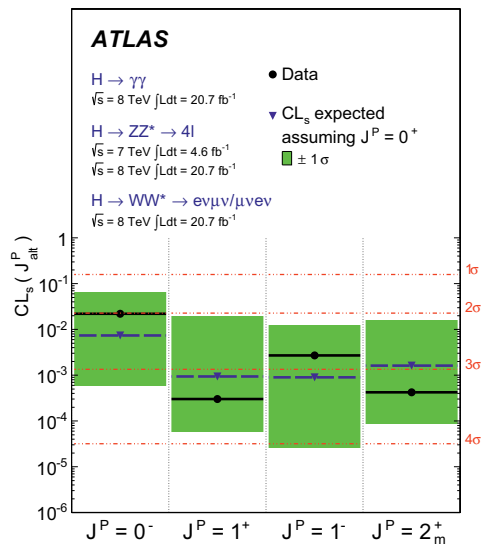

Figure 5. Expected (blue triangles/dashed lines) and observed (black circles/solid lines) confidence level $\mathrm{CL}_{\mathrm{s}}$ for alternative spin-parity hypotheses assuming a $0^{+}$signal.

decay channels. The reported results include measurements of the mass and signal strength, evidence for production through vector-boson fusion, constraints on couplings to bosons and fermions as well as on anomalous contributions to loop-induced processes, and the spin and parity studies provide evidence for the spin- 0 nature of the Higgs boson, with positive parity being strongly preferred. The precision exceeds previously published results in several cases. All measurements are consistent with expectations for the Standard Model Higgs boson.

\section{References}

[1] ATLAS Collaboration, Physics Letters B 716, 1-29 (2012)

[2] ATLAS Collaboration, Observation and study of the Higgs boson candidate in the two photon decay channel with the ATLAS detector at the LHC ATLAS-CONF-2012-168, 2-3 (2012)

[3] ATLAS Collaboration, Updated results and measurements of properties of the new Higgs-like particle in the four lepton decay channel with the ATLAS detector ATLAS-CONF-2012-169, 413 (2012)

[4] ATLAS Collaboration, Update of the $H \rightarrow W W^{*} \rightarrow \ell v \ell v$ analysis with $13.0 \mathrm{fb}^{-1}$ of $\sqrt{s}=8 \mathrm{TeV}$ data collected with the ATLAS detector ATLAS-CONF-2012-158, 4-7 (2012)

[5] ATLAS Collaboration, Physics Letters B 726, 120-144 (2013)

[6] Sara Bolognes and etc., On the spin and parity of a single-produced resonance at the LHC arXiv:1208.4018v3, (2014)

[7] R.J.Fries, Angular Distribution of Drell Yan Pairs in p+A at LHC arXiv:hep-ph/0211423v1, (2002) 